\title{
Ultrasound-modulated optical tomography using four-wave mixing in photorefractive polymers
}

Huiliang Zhang, Philip Hemmer, Peng Wang, Shuji Rokutanda, Michiharu Yamamoto, et al.

Huiliang Zhang, Philip Hemmer, Peng Wang, Shuji Rokutanda, Michiharu Yamamoto, Lihong V. Wang, "Ultrasound-modulated optical tomography using four-wave mixing in photorefractive polymers," Proc. SPIE 6856, Photons Plus Ultrasound: Imaging and Sensing 2008: The Ninth Conference on Biomedical Thermoacoustics, Optoacoustics, and Acousto-optics, 68561S (28 February 2008); doi: 10.1117/12.764987

SPIE. Event: SPIE BiOS, 2008, San Jose, California, United States 


\title{
Ultrasound-modulated optical tomography using four-wave mixing in photorefractive polymers
}

\author{
Huiliang Zhang, ${ }^{\text {a }}$ Philip Hemmer ${ }^{\mathrm{a}}$, Peng Wang ${ }^{\mathrm{b}}$, Shuji Rokutanda ${ }^{\mathrm{b}}$, \\ Michi Yamamoto ${ }^{\mathrm{b}}$, Lihong V. Wang ${ }^{\mathrm{c}}$ \\ ${ }^{a}$ Department of Electrical and Computer Engineering, Texas A\&M University, College Station, TX, \\ 77843; \\ ${ }^{b}$ Nitto Denko Technical, 501 Via Del Monte Rd, Oceanside, California 92054; \\ ${ }^{c}$ Optical Imaging Laboratory, Department of Biomedical Engineering, Washington University in \\ St. Louis, Campus Box 1097, One Brookings Dr. St. Louis, Missouri, 63130
}

\begin{abstract}
Ultrasound-modulated optical tomography uses a well focused ultrasound beam to modulate diffuse light inside soft biological tissues. This modality combines the advantages of ultrasound resolution with optical contrast. However, because of the low ultrasound modulation efficiency, the large background of un-modulated photons gives a low signalto-noise ratio. Here we report a technique for detection of ultrasound-modulated light using a phase conjugated signal generated by four-wave mixing in a photorefractive polymer. The experimental results demonstrate the potential of this method to detect ultrasound-modulated optical signals in a highly scattering media with an excellent signal-to-noise ratio.
\end{abstract}

Keywords: Ultrasound-modulated optical tomography, four wave mixing, photorefractive polymer, phase conjugate

\section{INTRODUCTION}

Optical imaging of soft biological tissue has become an increasingly interesting topic in biomedical research. Though pure optical imaging provides excellent optical contrast, it lacks good spatial resolution especially for the strong scattering present in a thick biological sample. Various imaging modalities have been proposed to overcome this difficulty, among which the ultrasound-modulated optical tomography (UOT) $)^{1,2}$ is a promising hybrid technique. In UOT, a focused ultrasound beam is used to phase modulate the diffusive light inside a soft biological tissue sample. Detection of the ultrasound-modulated (or tagged) light enables one to recover the spatial distribution of the diffusive photon fluence inside the sample, thus to reveal the distribution of optical properties, which are often of biomedical interests. This technique was first investigated by Marks et al. ${ }^{1}$ and has been studied by various groups. Wang et al. ${ }^{2}$ developed UOT by modulating a $c w$ laser light with a $c w$ ultrasound and obtained images of buried objects in tissuemimicking phantoms. This work was followed by Kempe et al. ${ }^{3}$ Leveque et al. ${ }^{4}$, who improved the signal to noise ratio (SNR) of UOT by a parallel speckle detection method, where a CCD camera was used as a detector array with a sourcesynchronized lock-in technique. Sakadzic and $\mathrm{Wang}^{5}$ used short ultrasound pulses to modulate the diffusive light and a long cavity Fabry-Perot interferometer filter was employed to obtain microscopic UOT images in biological tissues with a resolution scalable to the ultrasound wavelength $(<100 \mathrm{um})$ for an imaging depth deeper than $3 \mathrm{~mm}$. Murray et al. ${ }^{6}$ proposed the use of a two wave mixing (TWM) interferometer with a photorefractive(PR) crystal to enhance the

Photons Plus Ultrasound: Imaging and Sensing 2008: The Ninth Conference on Biomedical

Thermoacoustics, Optoacoustics, and Acousto-optics, edited by Alexander A. Oraevsky, Lihong V. Wang,

Proc. of SPIE Vol. 6856, 68561S, (2008) · 1605-7422/08/\$18 · doi: 10.1117/12.764987

Proc. of SPIE Vol. 6856 68561S-1 
detection efficiency. Xu et $a l^{7}$ uses TWM method to further distinguish optical properties and mechanical properties in soft biological tissues with UOT mechanism. A variation of their approach was implemented by Boccara et al. ${ }^{8}$ with a faster response PRC (GaAs) which works in the near infrared $(\lambda=1064 \mathrm{~nm})$. Theories on the mechanism of ultrasound modulation of scattered light were also developed by various groups. ${ }^{3,9,10}$

The low SNR of UOT, however, remains the biggest challenge for more practical imaging applications in biomedical research. The difficulty comes from several facts. One of the most important facts is that the part of diffusive light modulated by localized ultrasound inside the medium is overwhelmed by un-modulated, background diffuse light. For example in ref. 11, the dc value (background) is 30 times larger than ac amplitude (signal), so that a lot of averaging is needed to improve the SNR for imaging purpose. Therefore filtering out the background is extremely necessary for further improvement of UOT modality.

We report a novel technique for detection of the UOT signals by use of the four-wave mixing (FWM) instead of the TWM employed by all previous publications. FWM is implemented to generate phase conjugate waves. In this method, a nonlinear medium is pumped by a pair of counter-propagating laser beams with the same optical frequency. When a signal beam is incident into the medium, a fourth beam is generated. The fourth beam is propagating opposite to the signal beam and is a time-reversed replica of the signal beam. When the PR polymer is illuminated with a monochromatic reference wave, for example, $A_{1}(\omega)$ and a polychromatic signal wave $A_{2}=A_{2}(\omega)+A_{2}{ }^{\prime}\left(\omega^{\prime}\right)+A_{2}{ }^{\prime \prime}\left(\omega^{\prime \prime}\right)+\ldots$, only the component $A_{2}(\omega)$ will contribute to the formation of stable fringes. The components of $\mathrm{A}_{2}$ whose frequency differs from $\omega$ will only produce fringes having a rate of travel which is too high for the material to respond to. The counter-propagating laser beam (probe beam) only reads out the component $A_{2}(\omega)$. In this way, FWM in PR materials can work as a frequency filter.

FWM in PR crystals has been suggested for use as a frequency filter in the detection of ultrasound-modulated optical signals $^{13}$, but never demonstrated for UOT. A similar method with low frequency vibration, instead of high frequency ultrasound modulation, has been studied by Khoury et $a l^{14}$, but by making use of un-modulated light. However this method only applies to weakly scattering media such as diluted milk-water solution. Both these methods with whole plane imaging cannot work deep in highly scattering media. A related FWM technique, laser-based ultrasound for defect detection which involves reflection from vibrating surface, has been used to improve $\mathrm{SNR}^{15}$ by reducing bandwidth of the measurement compared with the time-domain method (for example TWM in ref 6). Considering the low SNR feature in UOT, four-wave mixing is quite a promising method for real application, because only ultrasound frequency modulated signal will be collected in the form of phase conjugate light. But for TWM frequency-domain detection ${ }^{7}$, the amplified frequency-matched UOT signal is collected along with the un-modulated light, which deteriorates the SNR so that further lock-in method is employed.

It is quite difficult to find proper PR crystals which have the proper phase conjugate reflectivity (defined as the ratio between the phase conjugate light and incoming signal light) and fast enough response time to match the speed of speckle de-correlation. PR polymers represent a comparatively new class of PR materials. These devices can offer very high internal diffraction efficiencies and have been demonstrated to exhibit response time on the order of milliseconds ${ }^{16}$. Furthermore, the fabrication procedures for these devices are significantly simpler than for PR crystals and can be done at low cost in a small-scale chemical laboratory.

\section{Experimental Setup}

The experimental setup can be seen in Fig. 1. Light from a 90-mW frequency-doubled Nd:YAG laser source was sent to a variable beam splitter, which was composed of a half wave plate and a polarizing beam splitter. The imaging was performed in a degenerate forward FWM geometry with the two writing beams (Fig.1, reference and signal light beam) subtending an angle around $40^{\circ}$ and interfering in the polymer to generate a hologram by the PR process. The polymer 
sample was tilted around $50^{\circ}$ with respect to the bisector of the two writing beams and was subject to an applied voltage of $7.5 \mathrm{kV}$. The hologram was read out by the probe beam, which was counter-propagating with the reference beam. For higher phase conjugate reflectivity, the writing and reading beams were $p$ polarized. Our phase conjugate detection system employed a PR polymer (from Nitto Denko Technical) with 100um in thickness. In this letter, the polymer composition was TPD:CBz:DCST 5:5:1 copolymer 50/ FDCST 30/ ECz 20. Under our experimental conditions, the response time of the PR polymer was approximately $300 \mathrm{~ms}$. The phase conjugate signal of modulated scattered light was collected by lenses 1 and 2, and fed in to a photodiode. The sound source was a spherically focused piezoelectric transducer (Ultran Lab VHP100-1-R38), with a 37.5-mm focal length and a center frequency of $1 \mathrm{MHz}$. The ultrasonic axis was perpendicular to the phantom illumination direction.

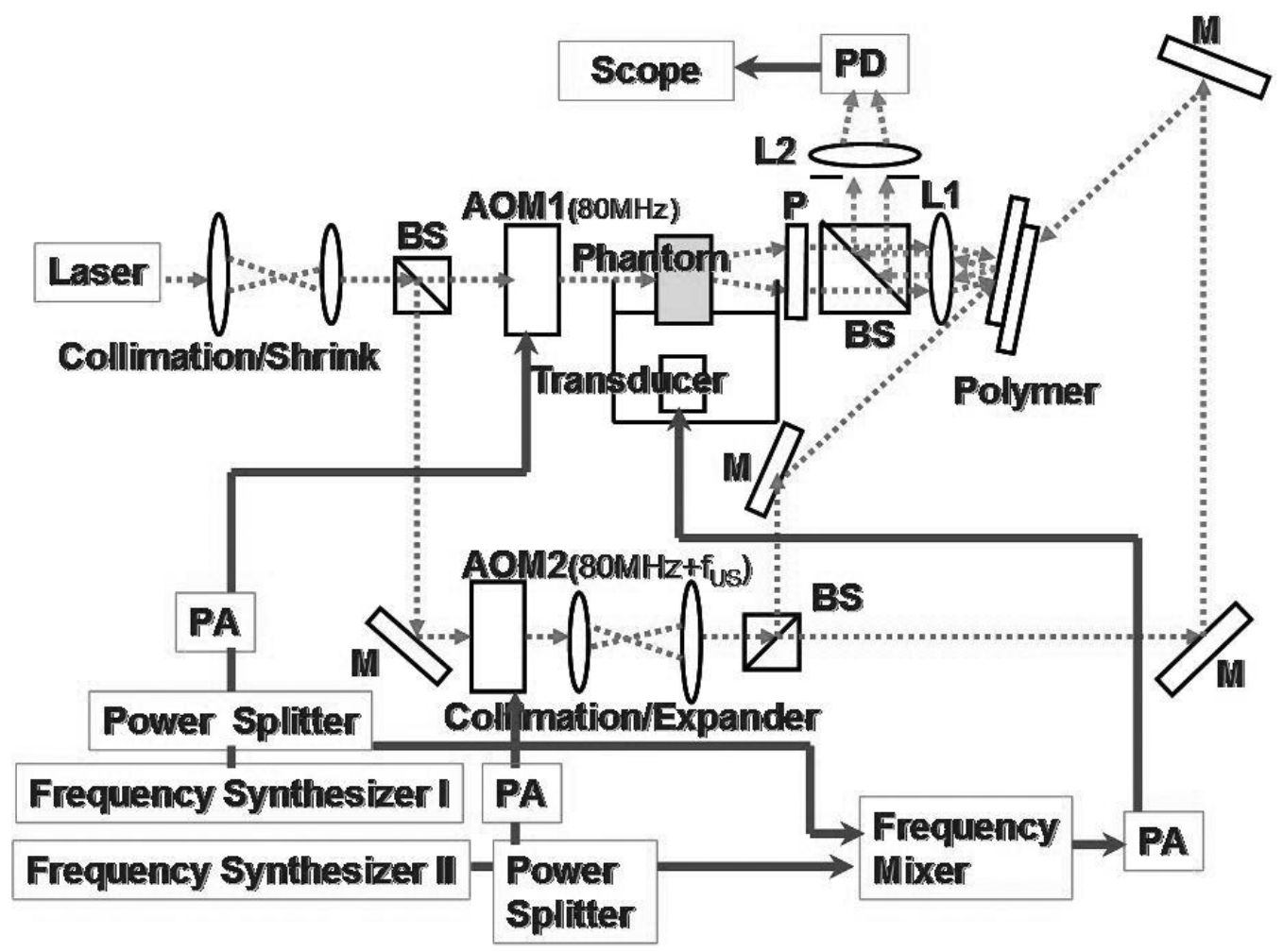

Figure. 1. Schematic of the experimental setup: BS, beam-splitter; AOM, acoustic-optics modulator; L, lens; PD, photo-diode; P, polarizer; PR polymer, photorefractive polymer; M mirror; PA, power amplifier; PS, power splitter; FS, frequency synthesizer; FM, frequency mixer. LP, low pass filter.

\section{Results}

To clarify the issue of angle acceptance, we designed an experiment for two wave mixing (TWM) which helped us to observe the gain change along with the angle difference. Setup is shown in as Fig. 2. 


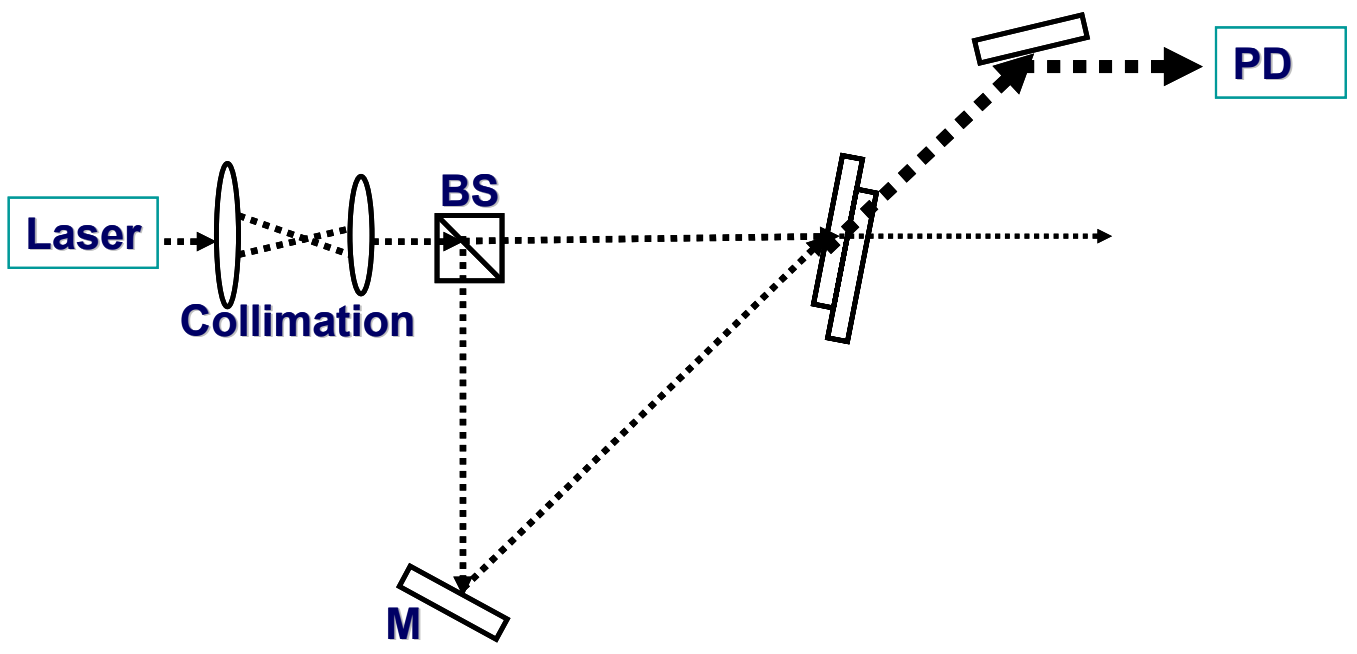

Figure. 2. Schematic of the TWM experimental setup: BS, beam-splitter; PD, photo-diode.

Light from laser is splitted into two parts: one part woks as the pump, the other works as the probe. Through TWM coupling effect, the energy of the pump will be transferred to the probe beam. We call the ratio between amplifier probe intensity to original probe intensity as the gain. The gain change reflects the angle acceptance (limited by photorefractive effect) of the material. We recorded the data and plotted the relationship as shown in Fig. 3. From this figure, we are sure that our material has angle acceptance as big as $\sim 50$ degrees. This value is usually much better than photorefractive crystals in the condition of the same response time.

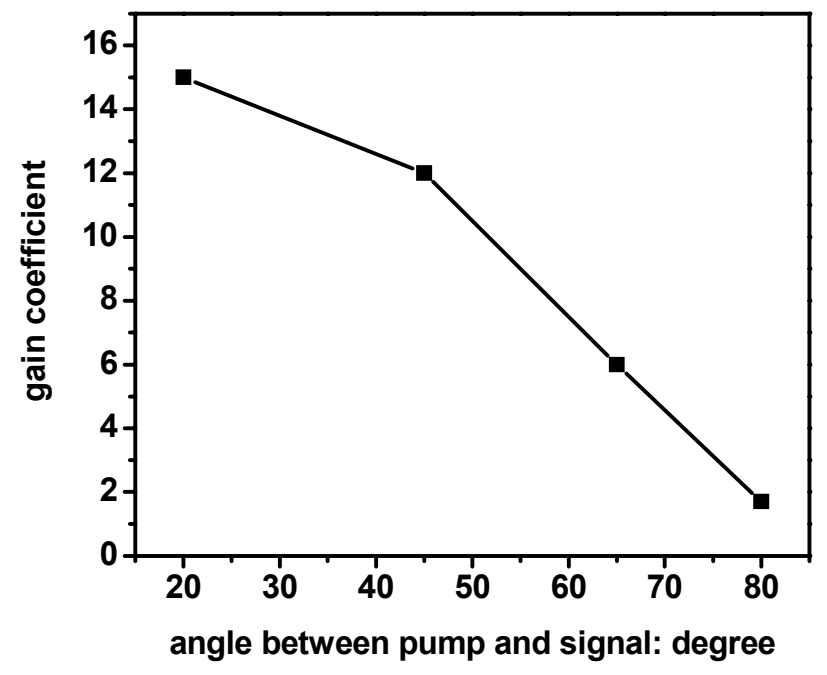

Figure. 3. Relationship between gain coefficient and suspension angle for TWM experiment

Figure 4 shows the signal intensity changes when ultrasound (with peak pressure around $0.7 \mathrm{MPa}$ ) is turned on and turned off. When ultrasound is off, no phase conjugate signal can be collected because the frequency of reference beams is different from that of signal beams, although there is still some residual light coming from scattering of the two reference beams. When ultrasound is turned on, a small component of the signal beam has the same frequency as the reference beam and phase conjugate light is generated. Because it takes time (PR response time) to write the holograph, 
the phase conjugate light increases gradually at first. After hundreds of milliseconds, the signal begins to stabilize, then UOT signal can be collected. To minimize the influence of background noise, subtract the averaged background. But still clearly the background has been suppressed a lot compared with heterodyne method ${ }^{10}$ and TWM interferometer by at least one order of magnitude. As mentioned before, phase conjugate reflectivity is quite critical in this application. In our case, the reflectivity can be more than unity. With a higher reflectivity polymer, higher signal to background ratio could be achieved.

A one dimension scan was performed (Figure. 5) to demonstrate the ability of FWM technique to detect a buried optical inhomogeneity (based on optical absorption). The tissue phantom consist of $10 \%$ porcine gelatin and $1 \%$ Intralipid concentration, resulting in a reduced scattering coefficient $\mu_{s}^{\prime}=10 \mathrm{~cm}^{-1}$. The outside dimension of the phantom sample was $6 \mathrm{~cm} \times 6 \mathrm{~cm} \times 1.5 \mathrm{~cm}$ and the phantom was oriented such that the laser passed through the $1.5-\mathrm{cm}$ thickness. One 0.2 $\mathrm{cm}$ thick (in the light propagation direction) Trypan-blue dyed object was buried in the phantom. In the scan direction, the objects are $0.3-\mathrm{cm}$ wide. The phantom was scanned in $0.5-\mathrm{mm}$ steps perpendicular to the light-propagation direction

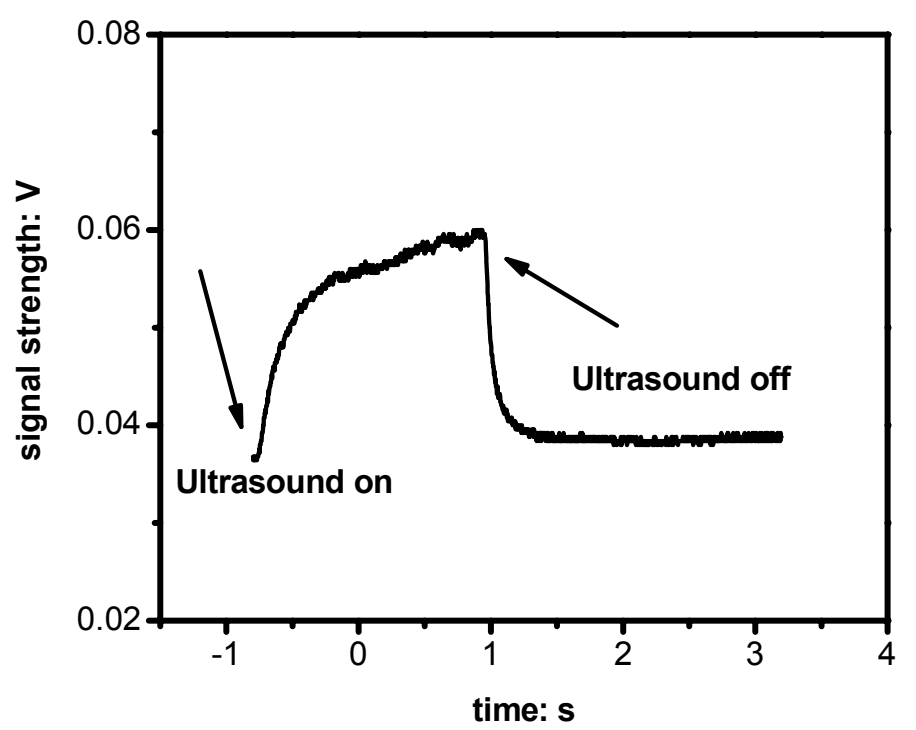

Figure. 4. Typical signal for UOT. Ultrasound is turned on. After several seconds, ultrasound is turned off.

and the ultrasound propagation direction. To achieve steady state for data collection, we took data after several seconds waiting time. In this case, we used low pass filter ( $25 \mathrm{~Hz}$ cut off frequency) to smooth out the high frequency noise. 


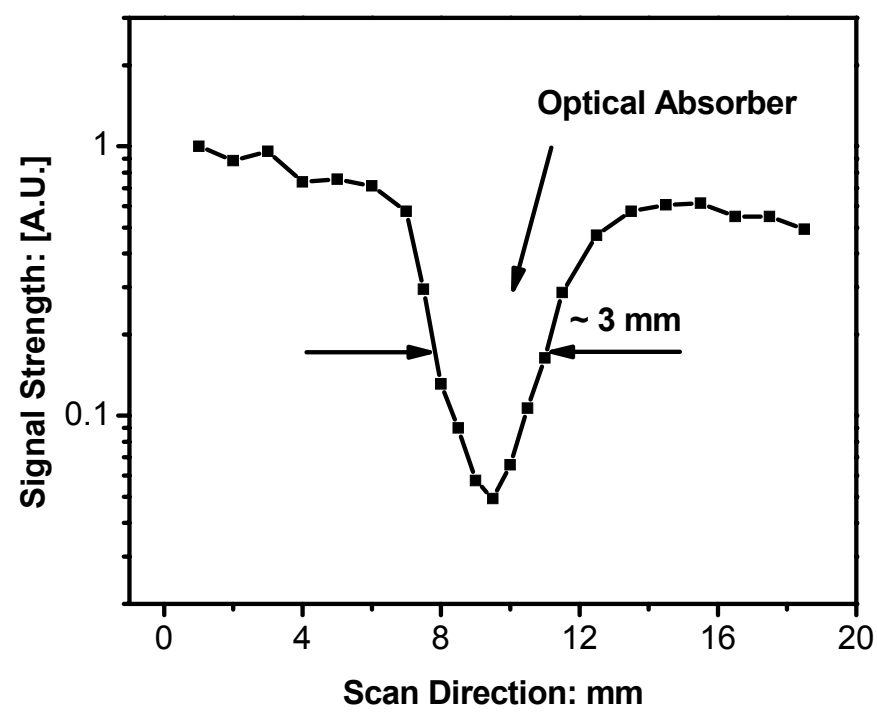

Figure. 5. Signal observed as the transducer is scanned across the tissue mimicking phantom

\section{Discussions and Conclusions}

The SNR issue is the greatest challenge for UOT. To increase SNR, we can either improve signal strength or decrease background (un-modulated photons) noise. For the point view of FWM, it has the obvious advantage of low background noise level.

To further improve the signal to background ratio, one possible approach is to use better performance materials. We had the experience of much better FWM reflectivity $(\sim 400 \%)$, for He-Ne $(632 \mathrm{~nm})$ laser. The sensitizer of the polymer is C60. Also it is better to use longer wavelength laser for deeper penetration in biological tissues ${ }^{17}$. The difficulty here is how to get the proper red laser which can supply hundred milli-watts for imaging purpose. It took us long time for our ring dye laser to work within proper wavelength and output power. More research in this method is still under investigation.

Another serious problem, which should be taken into consideration, is beam fanning. Beam fanning is an amplified scattering effect that occurs in high-gain PR organic materials (just as in inorganic crystals). Fanning manifests itself through an intensity loss as a single beam is transmitted through a sample with an electric field applied. Fanning is in a random direction because of its scattering nature. This will hurt our signal a lot, because both the phase-conjugate light and bean fanning are on the order of hundred nano-watts. It was found that to minimize the beam fanning, a negative high voltage has to be applied to the sample electrode at which the writing beams are incident. ${ }^{18}$ Also, reducing the beam diameter helps to minimize beam fanning ${ }^{19}$. However, at higher electric fields, even these measures are not sufficient to suppress the fanning ${ }^{20}$. In our case, usually we use voltage across the polymer for around $7 \sim 8 \mathrm{KV}$ because we need the big electrical field to enhance FWM efficiency. For 100um thickness polymer, the electrical field will be very high. How to optimize the trade-off between high voltage and beam fanning is still under investigation.

In summary, we have shown the feasibility of FWM for UOT as a nearly background free method. With PR polymers, the same level of background noise as the signal has been achieved. It should be mentioned that the use of thin PR 
layers is particularly important for this application because the angular bandwidth of the imaging system is inversely proportional to the interaction length, and a larger angular bandwidth will help optics alignment and suppress light scattering noise. PR polymers also possess structural flexibility that can lead to significantly improved efficiency by means of molecular engineering. With better performance polymer materials, it is possible to improve the signal to background ratio.

\section{Acknowledgement}

We appreciate Sava Sakadžic' for fruitful scientific discussions. This research was supported by the AFOSR FA955004-1-0247, National Institute of Health grant R33 CA 094267 and Nitto Denko Technical Corp.

\section{REFERENCES}

1 F. A. Marks, H. W. Tomlinson, and G. W. Brooksby, "A comprehensive approach to breast cancer detection using light: photon localization by ultrasound modulation and tissue characterization by spectral discrimination," Proc. SPIE. 1888, 500-510 (1993)

2 L. V. Wang, S. L. Jacques, and X. Zhao, "Continuous-wave ultrasonic modulation of scattered laser light to image objects in turbid media," Opt. Lett. 20, 629-631 (1995)

3 M. Kempe, M. Larionov, D. Zaslavsky, and A. Z. Genack, "Acousto-optic tomography with multiply scattered light," J. Opt. Soc. Am. A. 14, 1151-1158 (1997)

4 S. Leveque, A. C. Boccara, M. Lebec, and H. Saint-Jalmes, "Ultrasonic tagging of photon paths in scattering media: parallel speckle modulation processing," Opt. Lett. 24,181-183 (1999)

$5 \quad$ S. Sakadzic and L. V. Wang, "High resolution ultrasound-modulated optical tomography in biological tissues", Opt. Lett. 29, 2770-2772 (2004)

6 T. W. Murray, L. Sui, G. Maguluri, R. A. Roy, A. Nieva, F. Blonigen, and C. A. DiMarzio, "Detection of ultrasound modulated photons in diffuse media using the photorefractive effect," Opt. Lett. 29, 2509-2511, (2004)

$7 \quad \mathrm{X}$. Xu, H. Zhang, P. Hemmer, D. Qing, C. Kim and L. V. Wang, "Photorefractive detection of tissue optical and mechanical properties by ultrasound modulated optical tomography," Opt. Lett. 32, 656-658 (2007)

$8 \quad$ F. Ramaz, B.C. Forget, M. Atlan, A.C. Boccara, M. Gross, P. Delaye, G. Roosen, "Photorefractive detection of tagged photons in ultrasound modulated optical tomography of thick biological tissues," Opt. Express. 12, 5469-5474 (2004)

9 L. V. Wang, "Mechanisms of Ultrasonic Modulation of Multiply Scattered Coherent Light: An Analytic Model," Phys. Rev. Lett 66 (043903), 1-4, (2002)

10 S. Sakadzic and L. V. Wang, "Correlation Transfer and Diffusion of Ultrasound-Modulated Multiply Scattered Light," Phys. Rev. Lett 97(163902), 1-4, (2006)

${ }_{11}$ L. V. Wang and X. Zhao, "Ultrasound-modulated optical tomography of absorbing objects buried in dense tissue-simulating turbid media," Appl. Opt. 36, 7277-7282 (1997)

12 E. Bossy, L. Sui, T. W. Murray and R. A. Roy, Fusion of conventional ultrasound imaging and acousto-optic sensing by use of a standard pulsed-ultrasound scanner," Opt. Lett. 30, pp. 744-746 (2005)

13 D. Dolfi and F. Micheron, "Imaging process and system for transillumination with photon frequency marking," international patent WO 89/00278, January 12, 1989.

14 J. Khoury, J. S. Kane, P. D. Gianino, P. L. Hemmer and C. L. Woods, "Homodyne and heterodyne imaging through a scattering medium," Opt. Lett. 26, 1433-1435, (2001)

15 T. C. Hale and K. Telschow, "Optical lock-in vibration detection using photorefractive frequency domain processing," Appl. Phys. Lett. 69, 2632-2634 (1996)

16 For a recent review: O. Ostroverkhova and W. E. Moerner, "Organic Photorefractives: Mechanisms, Materials, and Applications," Chem. Rev. 104, 3267-3314 (2004)

17 V. V. Tuchin, Handbook of Optical Biomedical Diagnostics (SPIE Press, Bellingham, Wash, 2002)

18 A. Grunnet-Jepsen, C. L. Thompson, R. J. Twieg and W. E. Moerner, "Amplified scattering in a high-gain photorefractive polymer" J. Opt. Soc. Am. B 15, 901-904, 1998 
19 M. R. Leahy and D. J. McGee, "Influence of pump beam diameter on beam fanning in a doped photorefractive polymer composite", Opt. Commun. 187, 277-282 (2001).

20 O Ostroverkhova, U Gubler, D Wright, W. E. Moerner, M. He and R. Twieg, "Recent advances in understanding and development of photorefractive polymers and glasses", Adv. Function. Mater, 12, $621-.629$ (2002) 